\title{
DYSLIPIDEMIA AND DISRUPTION OF L-CARNITINE IN ALUMINUM EXPOSED WORKERS
}

\author{
By \\ ${ }^{1}$ Gaballa IF, ${ }^{1}$ Helal SF, ${ }^{2}$ Rashed LA and ${ }^{1}$ Abdelsamea AA \\ ${ }^{1}$ Department of Occupational and Environmental Medicine, \\ ${ }^{2}$ Department of Biochemistry Faculty of Medicine, Cairo University
}

\begin{abstract}
Introduction: Aluminum is the most widely used non-ferrous metal with suggested increase in the intra-cellular lipid accumulation. Aim: The aim of this work is to study the effect of Aluminum on lipid metabolism and plasma lipid profile giving special concern to the role of L-Carnitine in occupational Aluminum toxicity. Materials and Methods: The study was conducted on 40 exposed workers in an aluminum foundry compared to 40 non-exposed subjects. Detailed occupational and medical histories were taken with full clinical examination. Laboratory investigations in the form of liver enzymes, complete lipid profile, aluminum serum level and serum L-Carnitine were done for the whole population. Results: Clinical examination of studied population revealed the presence of higher frequency of hypertension, arrhythmias, ischemic heart disease, and stroke among workers exposed to aluminum when compared to non-exposed (statistical difference was significant, except for stroke). Serum aluminum level showed a high significant statistical elevation in group exposed to aluminum $(23.9 \pm 18.7)$ than the

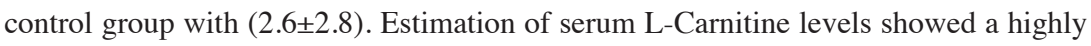
significant statistical difference between both groups with lower levels among exposed (7.0 \pm 3.3$)$ than non-exposed $(15.9 \pm 5.4)$ workers. Aluminum exposed workers showed an elevation in cholesterol (199.3 \pm 21.5$)$, triglycerides (86.218 \pm 7.113$)$, and low density lipoproteins $(142.5 \pm 22.8)$ compared to the control group with a highly significant statistical difference. The level of high density lipoproteins showed marked decrease (39.6 \pm 7.2$)$ among exposed than non-exposed workers with a difference that was also highly statistically significant. No statistically significant difference was found between both groups as regards liver enzymes in the form of aspartate transaminase and alanine transaminase. As for the different correlations with aluminum, no statistical significance
\end{abstract}


was obtained. Conclusion: Aluminum exposure among workers might be considered as a factor for disturbance in lipid profile levels with possible predisposition for the higher incidence of vascular impairment through direct effect on the serum L-Carnitine levels.

Recommendations: Periodic medical examination of workers exposed to aluminum in the aluminum industry should consider the routine measurement of lipid profile values. The use of L-Carnitine supplementation is to be recommended as effective measure in protecting exposed workers against aluminum-induced dyslipidemia.

Key Words: Aluminum, Lipid metabolism, Dyslipidemia, Hyperlipidemia, L-Carnitine.

\section{Introduction}

Aluminum is a metallic element with no known physiological role within the body. For many years, aluminum was not considered a toxic metal to humans even though older reports were associating the metal with several skeletal and neurological disorders suggesting aluminum exposure to be a predisposing factor for many health hazards (Nayak, 2002).

Aluminum toxicity was lately charged for the increase in the intracellular lipid accumulation as a result of induced disturbance in the oxidative-ATP production in the human liver cells. Consequently, those aluminum-stressed hepatocytes secrete lipids and proteins excessively (Mailloux et al., 2007). As such, aluminum-induced mitochondrial dysfunction promotes enhancement of lipogenesis with accumulation of the very low density lipoprotein secondary to decrease in the $\beta$-oxidation of fatty acids (Mailloux et al., 2011).
Recently, human astrocytes and hepatocytes exposed to aluminum that showed increased lipid accumulations were found to be characterized by lower levels of L-Carnitine. Accordingly, the amino acid L-Carnitine was suggested to be a critical metabolite indispensable for the metabolism of lipids as it facilitates fatty acid transportation into the mitochondria where $\beta$-oxidation takes place (Lemire et al., 2011).

\section{Aim of the work}

This study aims at (1) Detecting harmful effects of occupational aluminum exposure on lipid metabolism and lipid profile values. (2) Assessment of the role of L-Carnitine in dyslipidemia.

\section{Materials and Methods}

This cross sectional study was conducted in one of Egyptian Aluminum factories in Ain Helwan region. The study group consisted of 40 workers, ranging in age between 18 and 60 years 
(48.6 \pm 9.5$)$, working on the basis of $12 \mathrm{~h}$ /day with 1 day off, and working without any protective equipments during their working hours. The control group consisted of 40 unexposed healthy males matched with the study group for age (which ranged from 21 to 62 yrs (49.2 \pm 8.3$)$, sex, socioeconomic status, and smoking habits; they were selected randomly from the employers in the administration section of the factory at the same time of the research, which was away from the exposure zone. Participants in the study were volunteers; a written individual consent was obtained from every participant in the study either the exposed or the control group. These consents were reviewed by the ethical committee of Occupational Medicine and Environmental Diseases Department, Faculty of Medicine, Cairo University. The present study started after the approval of the ethical committee on the study protocol.

\section{Methods:}

(A) All workers were interviewed using a special questionnaire including occupational history; and full clinical examination. In ourstudy we detected stroke (as a manifestation of dyslipidemia) by patient's history of admission to hospital upon weakness of extremities, loss or diminution of sensation or dysarthia. Clinical examination was done helping to confirm diagnosis and detect residuals of stroke if present, neurological examination detecting motor power, sensations, cranial nerve affection, speech and gate. Both history and clinical examination revealed 5 stroke cases within exposed group, 4 of them in the form of hemiplegia the other one was monoplegia. Dysarthia accompanies 2 of hemiplegic cases with no residues. Non exposed group showed only 2 cases of monoplegia one of them with residual weakness in the left hand. IHD as a manifestation of heart disease was detected by history of hospital admission in ICU unit, previous catheterization (therapeutic) or ECG together with typical chest pain number of cases was 14 in exposed group to 4 in non exposed.

(B) Environmental monitoring of aluminum dust performed at the same time with our research in this factory revealed a range of aluminum dust levels extending 
from 0.33 to $3.4 \mathrm{mg} / \mathrm{m} 3$ in air (Siha et al., 2012). This level was found to be within the permissible limits of $5 \mathrm{mg} / \mathrm{m} 3$ for respirable Aluminum dust in air at the work place (OSHA, 2001).

(C) Laboratory investigations: From each subject $7 \mathrm{cc}$ of venous blood was taken through a venipuncture using a dry plastic syringe under aseptic conditions divided into two clean dry tube of which one was allowed to clot and then centrifuged to separate serum.

(1) Serum aluminum level: Serum aluminum was measured by graphite furanced atomic absorption spectrophotometry with zemman background (thermo elemental m-6 type)
(2) Detection of serum L-Carnitine

This assay employs the quantitative sandwich enzyme immunoassay technique.

(3) Measurement of lipid enzymes

Data analysis were done using SPSS statistical programmed version 16. Comparison between qualitative variables was done using the chi square test while the independent $\mathrm{T}$ test was used for the quantitative variables which were normally distributed. Nonparametric Kruskal-Wallis was done for the quantitative variables which were not normally distributed. Correlation was done to test for linear relations between variables. A P value less than 0.05 was considered of statistical significant, whereas less than 0.001 was considered of high statistical significance. 


\section{Results}

Table (1): Comparison between the exposed group to Aluminum and the control group as regard aluminum level, L- Carnitine level and lipid profile:

\begin{tabular}{|c|c|c|c|c|}
\hline & $\begin{array}{c}\text { Exposed group } \\
\text { No=40 }\end{array}$ & $\begin{array}{c}\text { Control group } \\
\text { No=40 }\end{array}$ & t Test & P value \\
\hline Cholesterol mean \pm SD & $199.3 \pm 21.5$ & $165.6 \pm 29.1$ & 27.616 & $<0.001$ ᄎ \\
\hline TG mean \pm SD & $86.218 \pm 7.113$ & $71.9 \pm 18.2$ & 38.902 & $<0.001$ ᄎ \\
\hline HDL mean \pm SD & $39.6 \pm 7.2$ & $49.3 \pm 5.6$ & 36.133 & $<0.001$ ᄎ \\
\hline LDL mean \pm SD & $142.5 \pm 22.8$ & $101.9 \pm 30.9$ & 29.769 & $<0.001$ * \\
\hline $\begin{array}{l}\text { Aluminum } \\
\text { Mean } \pm \text { SD }\end{array}$ & $23.9 \pm 18.7$ & $2.6 \pm 2.8$ & 28.863 & $<0.001$ ᄎ \\
\hline $\begin{array}{l}\text { L Carnitine } \\
\text { Mean } \pm \text { SD }\end{array}$ & $7.0 \pm 3.3$ & $15.9 \pm 5.4$ & 40.603 & $<0.001$ ᄎ \\
\hline
\end{tabular}

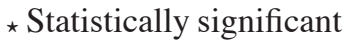

Table (1) shows high statistically significant difference between the exposed and control group as regards lipid profile values expressed in $\mu \mathrm{g} / 1$ with higher values among the exposed group [cholesterol mean (199.3 \pm 21.5$)$, TG mean $(86.218 \pm 7.11)$, LDL mean (142.5 \pm 22.8$)$ and HDL (39.6 \pm 7.2$)]$ than in controls [(165.6 \pm 29.1$)$, $(71.9 \pm 18.2),(101.9 \pm 30.9)$ and $(49.3 \pm 5.6)]$ respectively. The table shows that there is a highly statistically significant difference between exposed group and control one as regards serum aluminum and L- Carnitine levels. 
Table (2): Comparison between exposed and control groups as regards different complications of dyslipidemia [ischemic heart disease (IHD), stroke and hypertension (HTN)]:

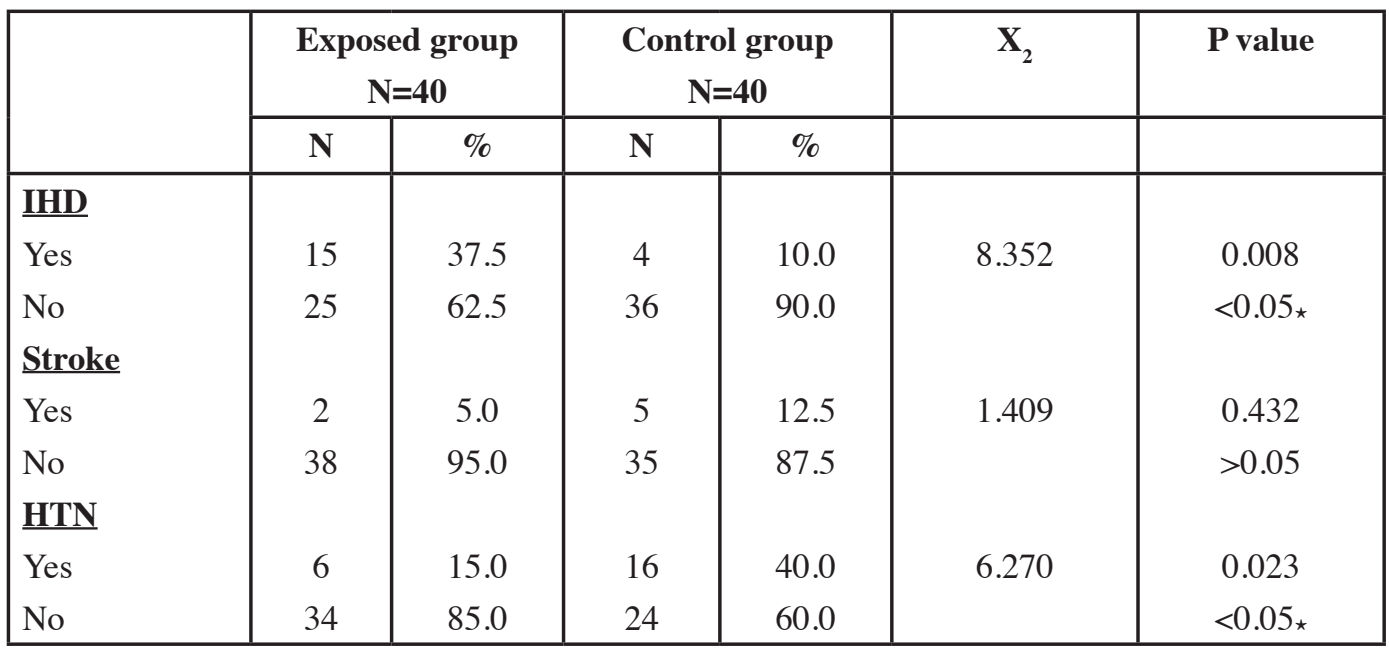

* Statistically significant

Table (2) shows that there was a statistically significant difference between exposed and control groups as regards IHD, hypertension but not stroke. 
Table (3): Comparison between smoker and non smoker exposed workers as regards lipid profile, $\mathrm{L}$-Carnitine and Aluminum levels:

\begin{tabular}{|l|c|c|c|c|}
\hline & $\begin{array}{c}\text { Smoker } \\
\mathbf{N}=\mathbf{2 1}\end{array}$ & $\begin{array}{c}\text { Non smoker } \\
\mathbf{N = 1 9}\end{array}$ & $\mathbf{t}$ Test & P value \\
\hline Cholesterol mean \pm SD & $200.9 \pm 22.6$ & $197.5 \pm 20.7$ & 0.496 & 0.481 \\
& & & & 0.05 \\
\hline TG & $86.2 \pm 7.5$ & $86.2 \pm 6.9$ & 0.535 & 0.465 \\
mean \pm SD & & & & $>0.05$ \\
\hline HDL \\
mean \pm SD & $40.9 \pm 4.3$ & $38.1 \pm 9.4$ & 0.728 & 0.393 \\
\hline $\begin{array}{l}\text { LDL } \\
\text { mean } \pm \text { SD }\end{array}$ & $142.8 \pm 23.9$ & $142.1 \pm 22.4$ & 0.031 & 0.05 \\
\hline $\begin{array}{l}\text { L-Carnitine } \\
\text { mean } \pm \text { SD }\end{array}$ & $6.8 \pm 3.7$ & $7.2 \pm 3.0$ & 0.728 & $>0.05$ \\
\hline $\begin{array}{l}\text { Aluminum } \\
\text { mean } \pm \text { SD }\end{array}$ & $23.9 \pm 19.4$ & $24.0 \pm 18.5$ & 0.003 & $>0.05$ \\
\hline
\end{tabular}

Table (3) shows no statistically significant difference between smokers and nonsmokers as regards plasma lipid profile, serum L- Carnitine or Aluminum levels. 
Table (4): Correlation coefficient between Aluminum levels to lipid profile values among exposed workers:

\begin{tabular}{|l|c|c|}
\hline & \multicolumn{2}{|c|}{ Aluminum level } \\
\hline Cholesterol & $\mathbf{r}$ & Pvalue \\
& -0.112 & 0.493 \\
TG & & $>0.05$ \\
\hline HDL & -0.286 & 0.073 \\
& & $>0.05$ \\
\hline LDL & 0.042 & 0.798 \\
& & $>0.05$ \\
\hline
\end{tabular}

Table (4) shows no statistically significant correlation between the levels of serum Aluminum to lipid profile among exposed workers.

Table (5): Correlation coefficient between serum Aluminum level and

L-Carnitine levels among workers exposed to Aluminum $(n=40)$.

\begin{tabular}{|l|c|c|}
\hline & & Aluminum \\
\hline \multirow{2}{*}{ L-Carnitine } & r value & -0.240 \\
\cline { 2 - 3 } & Pvalue & NS \\
\hline
\end{tabular}

Aluminum $(\mu \mathrm{g} / \mathrm{l})$, L-Carnitine ( $\mathrm{mmol} / \mathrm{l})$,

NS: Non significant $\mathrm{P}$ value $<0.05$

Table (5) shows no statistically significant correlation between the levels of serum Aluminum and L-Carnitine among exposed workers. 


\section{Discussion}

Aluminum is accused of causing multisystem toxicity as it has no evident physiological role in the human body (Nayak, 2002). At the workplace, the individuals can be subjected to high aluminum levels through inhalation of aluminum dusts and fumes in contaminated air (Yokel et al., 2008). Aluminum is proved to cause disturbance in lipid metabolism with a resultant dyslipidemia (Beladnoira et al., 2013). Dyslipidemia itself is an asymptomatic disorder, but over a period of decades, chronically elevated serum lipids were found to contribute to the formation of atheromatous plaques in overall arteries causing progressive stenosis or even complete occlusion of involved arteries (Finn et al., 2010).

In the current study, history and clinical examination revealed the presence of stroke as a manifestation of vascular impairment among 5 workers exposed to aluminum.

Surprisingly, two cases of monoplegia were noticed among the non-exposed group. Since our study group was small, the findings obtained were considered only suggestive as shown in (table 2).
Additionally,mostof studiesworking on CNS toxic manifestations related to long term exposure to aluminum reported slight impairment of memory functions, learning problems, attention deficit and depression corresponding to serum and urinary aluminum (Riihimäki et al., 2000). There was no unequivocal evidence of CNS toxicity from aluminum in the occupational setting supported by dose-response or doseeffect data over a relevant range of body burdens. In addition, no studies were found to be involved with aluminuminduced cerebrovascular impairment. Aluminum was reported to decrease the activity of (Ca+Mg)-ATPase, key enzyme involved in myocardial calcium transport. However, aluminum alone had no such effect, but its toxicity might potentiate the adverse effects of other stressful conditions or diseases on calcium regulatory myocardial protein calmodulin activity that may contribute to reduced sarcoplasmic reticulum calcium uptake (Levine et al., 1990). Many cases of arrhythmias, similar to our findings, have been recently reported among aluminum workers, thus suggesting the presence of toxic effects exerted by the metal on heart and blood vessels (Yildiz et al., 2012). 
European Economic Community (EEC), aware of aluminum related toxic manifestations, adopted a resolution, June 1986 suggesting that serum aluminum should be monitored quarterly. Aluminum levels of $60 \mu \mathrm{g} / \mathrm{L}$ or more were then considered indicator of excessive build-up of the aluminum body burden (Costantini et al., 1989). Following many researches relating aluminum at different levels with its toxic manifestations, serum aluminum concentration was then suggested to be a best indicator having a direct relation to the loading capacity of body aluminum and direct indicator for toxicity (Guo et al., 2002). Estimation to the levels of aluminum in serum among the studied population showed highly significant statistical difference between both groups with higher values among the exposed group as shown in (table 1). Similarly, other researchers reported high levels of aluminum in the body among the exposed workers whose duration of employment ranged between 5 and 32 years (mean=17.8, SD=6.7 years) (Sińczuk et al., 2006). The serum levels reported among electrolytic workers in related studies showed a significant statistical difference between those exposed $(25.18 \pm 2.65 \mu \mathrm{g} / \mathrm{L})$ and control $(9.97 \pm 2.83 \mu \mathrm{g} / \mathrm{L})$ groups
(Lu et al., 2013). Serum aluminum of $10 \mu \mathrm{g} / \mathrm{l}$ was considered a cut off level for health effects (Polizzi et al., 2002). This clearly explains the absence of any clinical manifestations related to serum aluminum among the nonexposed population with levels almost not exceeding the limit $(0-10.5 \mu \mathrm{g} / \mathrm{l})$.

It was postulated that the leaves of tobacco plants used to prepare cigarettes or other smoked products vary markedly in their constituents. As the tobacco plant is well known to absorb trace elements from the soil and to accumulate them in its leaves on large scale, the composition of tobacco is multifarious and appears in different formulations. Accordingly, the quantity of trace elements in tobacco might depend upon plant genotype, type of water, soil and their respective $\mathrm{pH}$, applied fertilizers or pesticides, and the environment (Csalari and Szantai, 2002). Among all elements, aluminum was considered second major trace element after $\mathrm{Fe}$ in all smoked tobacco products, and found in a range of 150 $2100 \mu \mathrm{g} / \mathrm{g}$ (Musharraf et al., 2012). Further analysis to the role of tobacco smoking in aluminum toxicity among our exposed workers failed to detect any significant statistical difference 
between smoker and non-smoker groups as shown in (table 3 ). These results were similar to the results of Nogaj and co-workers in (2007), who concluded that aluminum content in individuals exposed to tobacco smoking is much lower than non-exposed. They endorsed this finding to the presence of repeating co-dependences between aluminum and other metals as $\mathrm{Fe}, \mathrm{Mg}$ and $\mathrm{Zn}$ present in tobacco smoke.

Our work studied the relation between Aluminum exposure and lipid profile as shown in ( Table 4) .Aluminum was known to perturb the oxidativeATP production in human hepatocytes. This aluminum-induced mitochondrial dysfunction was reported to promote accumulation of more cholesterol and lipids. Accordingly, a metabolic profile favoring lipid production as well as increased activities and expression of certain lipogenic enzymes in hepatocytes exposed to aluminum was reported (Mailloux et al., 2007). Marked elevation of serum total cholesterol, low density lipoproteins and triglycerides in aluminum workers were demonstrated confirming presence of dyslipidemia as one of the occupational hazards to aluminum exposure (Shapovalova et al., 2010). These findings were also supported by other studies which suggested that human astrocytes and hepatocytes exposed to aluminum were characterized by increased lipid accumulation (Lemire et al., 2011). In fact, aluminum was shown to exert its effects on metabolism by disrupting lipid membrane fluidity and ending in oxidative stress causing intracellular lipid accumulation as a result of enhanced lipogenesis and decreased $\beta$ oxidation of fatty acids (Mailloux et al., 2011). The presence of hypercholesterolemia and hypertriglyceridemia among workers following aluminum exposure and intoxication was strongly suggested (Belaïd-Nouira et al., 2013).

Similarly, the present research showed a highly statistically significant difference between the exposed and control group as regards the different values of lipid profile favoring a state of hyperlipidemia among exposed workers with elevation of cholesterol, low density lipoproteins (LDL) and triglycerides compared to marked decrease in the levels of high density lipoproteins (HDL) among exposed workers as shown in (table 1), but we demonstrated also that there was no statistically significant correlation between the levels of serum Aluminum 
to lipid profile among workers exposed to Aluminum as shown in (table 4) and this is may be due to small size population examined. The relationship between serum Aluminum and different lipids showed an inversely proportional relation with the levels of cholesterol, LDL and triglycerides, but a direct relation with HDL levels. Even though such correlations are all insignificant, yet, they might confirm the impact of Aluminum exposure on inducing dyslipidemia.

Studies reported that human hepatocytes exposed to Aluminum had lower levels of L-Carnitine with diminished lipid oxidation. This was related to the sharp reduction in the response of the enzymes mediating the biogenesis of L-Carnitine to the Aluminum challenge (Lemire et al., 2011). In the current study, an inversely proportional relation was found between the level of serum Aluminum and L-Carnitine. However, the study failed to detect a significant correlation as shown in (table 5) suggesting that Aluminum-induced reduction in serum L-Carnitine level is not dose-dependent.

Other studies as well showed lowered levels of L-Carnitine in human hepatocytes exposed to Aluminum with diminished $\beta$-oxidation, increased lipid accumulation and concomitant reduction in reactive oxygen species (ROS) (Lemire et al., 2011).

\section{Conclusion}

Enhanced secretion of cholesterol, low-density lipoprotein and triglyceride may indicate a possible link between aluminum toxicity and dyslipidemia as Aluminum-exposed workers showed symptoms of vascular impairement caused by abnormal lipid accumulation. It was postulated that the disturbance is taking place through diminished L-Carnitine responsible for facilitating lipid entry to the mitochondria where oxidation occurs.

\section{Recommendations}

Werecommend the useofL-Carnitine as a biomarker for early lipid profile disturbance. Regular periodic medical examination of exposed workers should consider estimation to the levels of Aluminum in serum or even in urine either on a yearly basis or every other year as a monitor of the body burden. A level of $10 \mu \mathrm{g} / 1$ should be considered as a cut off level for serum Aluminum in Aluminum foundry workers. Environmental periodic air sampling and analysis for Aluminum metal in 
the workplace ambient air should be planned. Motivation of workers for the use of personal protective equipments as face masks or respirators. Though smoking was not proved a confounding factor, yet cessation campaigns should be regarded essential for all workers.

\section{References}

1. Belaid-NouiraY ,Bakhta H , Haouas Z , Slim IF, Neffati F ,Najjar M F and BenCheikh H (2013): Fenugreek seeds, a hepato-protector forage crop against chronic $\mathrm{AlCl} 3$ toxicity; $\mathrm{BMC}$ Vet. Res; 10:9-12

2. CostantiniS, Giordano R, Vernillo I ,Piccioni A and Zapponi G A (1989): Predictive value of serum aluminum levels for bone accumulation in haemodialized patients. Ann. Ist. Super. Sanita; 25(3):457-462.

3. Csalari Jand Szantai K(2002): Transfer rate of cadmium, lead, zinc and iron from the tobaccocut of the most popular Hungarian cigarette brands to the combustion products. Acta Aliment; 31:279-288.

4. Finn A V, Nakano M ,Narula J ,Kolodgie F D and Virmani R(2010): Concept of vulnerable/ unstable plaque. Arterioscler.Thromb.Vasc. Biol; 30(7):1282-1292.

5. GuoZ, Zhu Q, Hu C and Yang Y (2002): Study on lipid peroxidation of electrolyzing-aluminum workers. Wei. Sheng.Yan Jiu; 31(2):78-80.

6. LemireJ, Mailbox R, Darwich R , Auger C and Appanna V D (2011): The disruption of L-Carnitine metabolism by aluminum toxicity and oxidative stress; Toxicol.Let. J; 203(3):21926.

7. Levine S N, Sonnier G B and Abreo K (1990): Effects of diabetes mellitus and aluminum toxicity on myocardial calcium transport. Toxicol; 65(1-2):137-48.
8. Lu X T, Liang R F, Jia Z J, Wang H, Song WF, Li QY and Niu Q (2013): Effect of aluminum exposure on cognitive function in electrolytic workers and its influential factors. Zhon. Lao Dong Wei Sheng Zhi Ye Bing ZaZhi; 31(2):113-116.

9. Mailloux R, Lemire J, and Appanna V. (2007): Aluminum-induced mitochondrial dysfunction leads to lipid accumulation in human hepatocytes: a link to obesity. Cell Physiol. Biochem; 20(5):627-638.

10. Mailloux R J, Lemire J and Appanna VD (2011): Hepatic response to aluminum toxicity: Dyslipidemia and liver diseases. Exp. Cell. Res; 317(16):2231-2238.

11. Musharraf S G, Shoaib M, Siddiqui AJ ,Najamul-Haq M and Ahmed A(2012): Quantitative analysis of some important metals and metalloids in tobacco products by inductively coupled plasma-mass spectrometry (ICP-MS). Chem. Cent. J; 6:56-63.

12. Nayak P (2002): Aluminum: impacts \& diseases. Environ. Res; 89:101-115.

13. Nogaj E, Kwapuliński J, Misiołek M, Fisher A, Brodziak-Dopierała B, Misiołek H, Namysłowski G and Bogunia M (2007): Influence of passive smoking on content of Aluminium in pharyngeal tonsils from children living in Southern Poland. Przegl. Lek; 64(10):713-6.

14. OSHA (Occupational Safety and Health Administration) (2001): Aluminum oxide in the workplace atmosphere. Available at http:// www.osha.gov/dts/slts/methods/inorganic/ id198.html.

15. Polizzi S , Pira E, Ferrara M ,Bugiani M ,Papaleo A ,Albera R and Palmi S (2002): Neurotoxic effects of Aluminium among foundry workers and Alzheimer's disease. Neurotoxicol; 23(6):761-74.

16. Riihimäki V, Hänninen $H$,Akila $R$, Kovala $T$, Kuosma E ,Paakkulainen H, Valkonen S , and 
Engström B (2000): Body burden of aluminum in relation to central nervous system function among metal inert-gas welders. Scand. J. Work Environ. Health; 26(2):118-130.

17. Shapovalova VP, Ryzhova TV and Ryzhov VM (2010): Lipid metabolism state under exposure to noise and aluminum dust. Med. Tr. Prom. Ekol; 7:18-20.

18. Siha MS, Shaker DA and Ramadan MA (2012): Effect of occupational exposure to $\mathrm{Al}$ on parathyroid hormone and Calcium metabolism (thesis for master degree department of occupational and environmental medecine. chapter 5 (Results). 60-71.
19. Sińczuk-Walczak H ,Szymczak M , Aniołczyk H , Brzeźnicki S ,Raźniewska G ,TrzcinkaOchocka M and Matczak W (2006): The effect of combined exposure to chemical and physical factors on the nervous system in aluminum production: preliminary finding. Med. Pr; 57(1):7-13.

20. YildizM , Kocabay G and Ozkan M (2012): Aluminium-induced ventricular tachycardia. Am. J. Emerg. Med; 30(1):262.e1-2.

21. Yokel R A, Hicks C L and Florence R L (2008): Aluminum bioavailability from basic sodium aluminum phosphate, an approved food additive emulsifying agent, incorporated in cheese. Food Chem. Toxicol; 46:2261-2266. 